\title{
Chemical and Reconstruction-Induced Surface Core-Level Shifts: H on Low-Index W Surfaces
}

\author{
D. M. Riffe, G. K. Wertheim, and P. H. Citrin \\ AT\& T Bell Laboratories, Murray Hill, New Jersey 07974 \\ (Received 20 December 1989)
}

\begin{abstract}
The $\mathrm{H}$-induced shift of the surface-atom core-level binding energy in $\mathrm{W}(110)$ is shown to arise from two distinct effects, one chemical in nature and the other structural. The structural shift supports a recently proposed $p(1 \times 1)$ reconstruction that turns on at $\sim 0.5$ monolayer coverage. These new findings are used to provide a self-consistent interpretation of previously reported shifts from H-covered W(111) and $\mathrm{W}(100)$ surfaces.
\end{abstract}

PACS numbers: 79.60.-i, 68.35.Bs, 82.65.My

The inherently different electronic and structural environments of bulk and clean surface atoms is known to give rise to a shift in their core-electron binding energies. ' These surface core-level shifts (SCS's) have been observed to change in a variety of chemisorption systems, ${ }^{1,2}$ with the changes generally being attributed to the transfer of charge between the adsorbate and substrate in forming a chemical bond. However, when the process of chemisorption is accompanied by substrate reconstruction, which is often the case, this description no longer applies because the SCS's are affected not only by the adsorbate-substrate bonding but also by the reconstruction-induced change in the surface-atom coordination number. ${ }^{\prime}$ In such circumstances it would appear difficult, if not impossible, to distinguish between these two different but coexisting contributions to the change in the SCS.

Here we report on high-resolution core-level photoemission data from $\mathrm{W}(110)$ as a function of $\mathrm{H}$ coverage and show that the SCS change can indeed be separated into chemical and reconstruction-induced contributions. In light of these new findings, previously measured SCS values from the clean and $\mathrm{H}$-covered $\mathrm{W}(111)$ and W(100) surfaces are reinterpreted. ${ }^{3-5}$ Our analysis indicates that despite different $\mathrm{H}$ adsorption geometries ${ }^{6-8}$ the chemical shift for all three low-index W surfaces is essentially the same, and that despite different reconstructions of the (100) and (110) surfaces ${ }^{8,9}$ the structurally induced shifts are remarkably similar. The present work provides a unified picture for describing the effects of chemisorption in dissimilar systems and suggests that such an approach may also be applicable to other surfaces.

The photoemission experiments were performed using the AT\&T Bell Laboratories 6-m toroidal-gratingmonochromator beam line at the National Synchrotron Light Source. All data were measured with $70-\mathrm{eV}$ photons and 2-eV analyzer pass energy, giving a total instrumental resolution of $-85 \mathrm{meV} .{ }^{10}$ The single-crystal $\mathrm{W}$ ribbon was cleaned using standard procedures in a $\mathrm{H}_{2-}$ dominated vacuum of $\sim 1 \times 10^{-10}$ Torr. Sequential $\mathrm{H}_{2}$ doses, corrected for ambient exposure during data collection, were used to minimize systematic errors in deter- mining the absolute $\mathrm{H}$ coverages. ${ }^{11}$

A typical data set showing the influence of chemisorbed H on the SCS's is shown in Fig. 1. The unexposed surface shows two well-separated peaks, one due to bulk atoms at 31.4-eV binding energy and another due to (110) surface atoms shifted by $-321 \mathrm{meV}$ (subsurface contributions are not resolved for this close-packed surface ${ }^{10}$ ). The binding energy of the surface line increases with $H$ coverage $\Theta$ until it merges with the bulk at a saturation coverage of 1 atomic monolayer.

To quantify the change in SCS over the whole coverage range, some method of data analysis must be employed. Traditional analytical models of chemisorption provide an additional line for each inequivalent adsorbate-coordinated substrate atom and assume that these lines have identical shape and fixed binding energy. Such models, however, do not adequately represent the

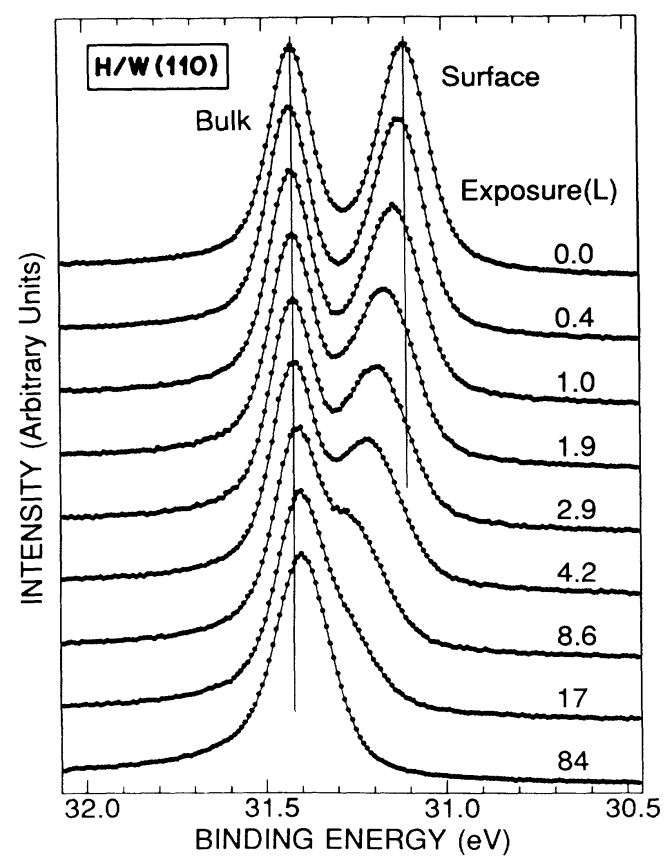

FIG. 1. Photoemission spectra of $\mathbf{W} 4 f_{7 / 2}$ electrons for increasing exposures of $\mathrm{H}$ in langmuirs $\left(1 \mathrm{~L}=10^{-6}\right.$ Torrsec $)$. 
physical situation for $\mathrm{H}$ on $\mathrm{W}$ for several reasons. First, in the presence of adsorbate-induced reconstruction the coordination of the $\mathrm{W}$ surface atoms to $\mathrm{W}$ as well as to $\mathrm{H}$ changes. Second, since the $4 f$ line shape in $\mathrm{W}$ has been shown to depend sensitively on the valence-band charge-density distribution, ${ }^{10}$ the $\mathrm{H}$-perturbed atoms cannot be assumed to have the same line shape as their unperturbed counterparts. Finally, as a practical matter, it is difficult to justify the use of multiple H-perturbed lines when none are resolved; e.g., see Fig. 1. In view of these facts, we have adopted a different approach. Only three components are used: one for the bulk, one for the unperturbed surface atoms, and one for the remaining surface atoms, which are influenced by $\mathrm{H}$. For the bulk

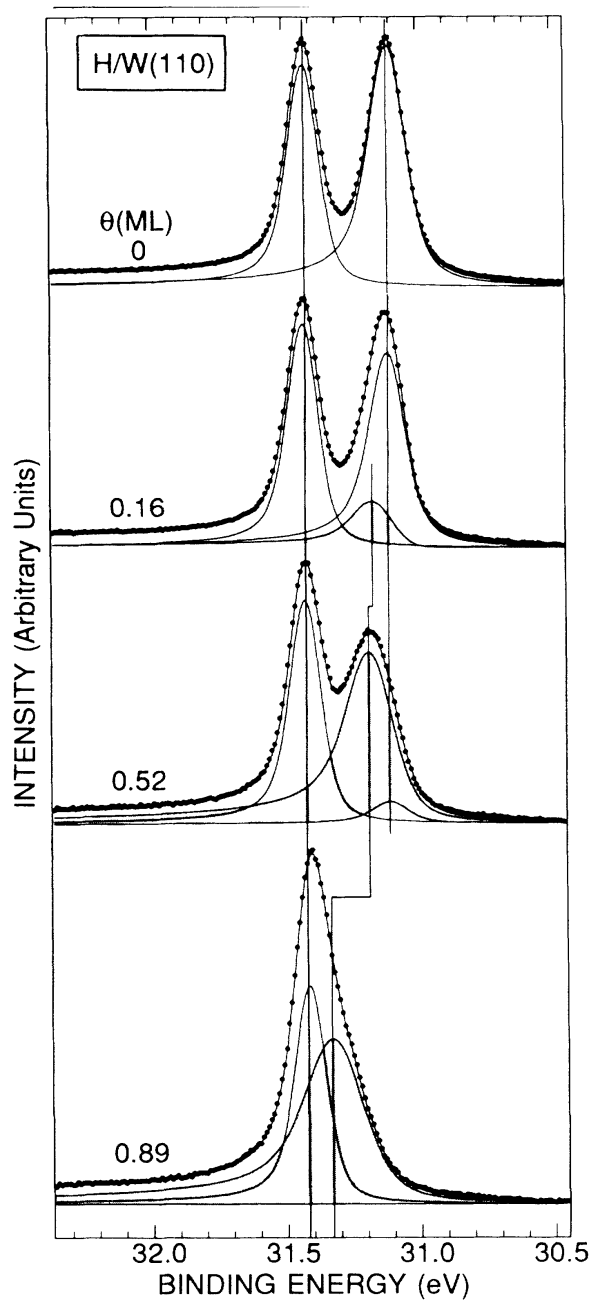

FIG. 2. Three-peak fit of $\mathrm{W} 4 f_{7 / 2}$ photoemission spectra for selected $\mathrm{H}$ coverages $\boldsymbol{\theta}$ in monolayers. Intensity of the bulk component at highest binding energy is shown equal for all fits. Bulk and unperturbed-surface line shapes and positions are held constant to their clean-surface values (see Ref. 10), but their amplitudes are allowed to vary with $\Theta$. Third component (shaded), describing the effect of the $\mathrm{H}$, is unconstrained. Solid circles are the data and the solid line is the fit. and unperturbed surface atoms we use the relative energy positions and line-shape parameters obtained from the clean surface ${ }^{10}$ as coverage-independent input; only the intensities are allowed to vary with $\Theta$. For the $\mathrm{H}$ influenced component, on the other hand, the line shape, position, and intensity are all left unconstrained because the number of $\mathrm{W}$ surface atoms and the degree to which they are chemically and structurally modified by $\mathrm{H}$ are all coverage dependent.

The results of fitting the data with these criteria are shown in Fig. 2. The three-peak analysis is seen to describe the data very well over a wide range of $\mathrm{H}$ coverages. It also reveals that at $\Theta=0.56 \pm 0.03$ monolayer (ML) the unperturbed-surface intensity vanishes. Since at coverages below $0.5 \mathrm{ML}$ the shift of the $\mathrm{H}$-perturbed component is small compared to the linewidth, its strongly correlated position and amplitude are difficult to determine accurately. The average SCS of all the surface atoms, $\bar{E}_{s}$, does, however, provide a reliable measure of the effect of the adsorbed $\mathrm{H}$. For coverages above 0.5 $\mathrm{ML}$, where the unperturbed component has vanished, our model gives $\bar{E}_{s}$ directly.

In Fig. 3 we have plotted the average SCS value as a function of coverage for $\Theta$ between 0 and 1.0 ML (also shown is the intensity of the $4-\mathrm{eV}$ zone-center peak

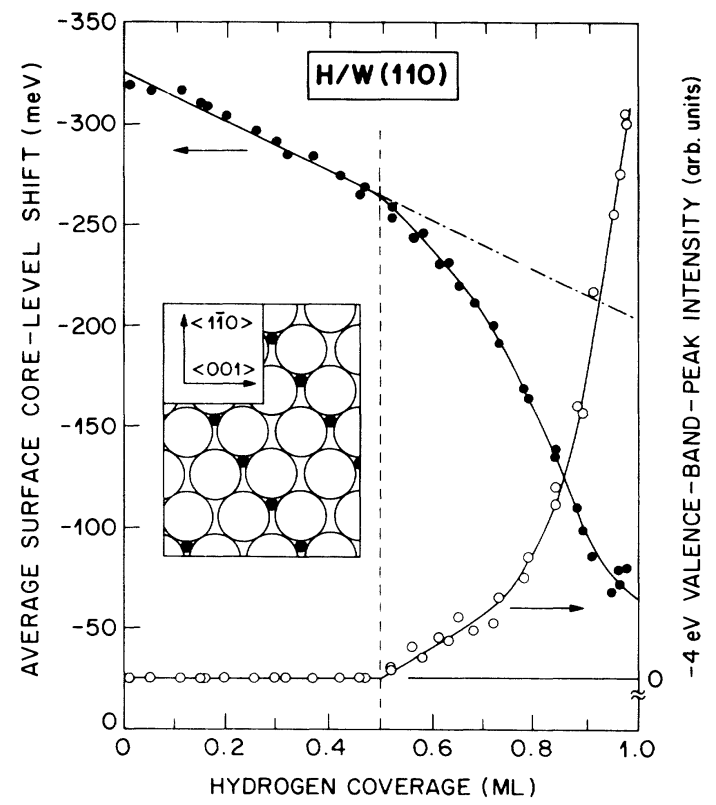

FIG. 3. Average SCS, $\bar{E}_{s}(\Theta)$ (solid circles), and $-4-\mathrm{eV}$ valence-band (VB) peak intensity (open circles) vs $\mathrm{H}$ coverage. Solid lines are drawn as guides to the eye. The dot-dashed line is an extrapolation of the solid line through the data. The dashed vertical line is drawn to contrast the different coverage dependences of the average SCS below and above $0.5 \mathrm{ML}$. Onset of $-4-\mathrm{eV}$ VB peak indicates $0.5-\mathrm{ML}$ coverage (see Refs. 11 and 15$)$. Inset: $\mathrm{W}(110) p(2 \times 1)-\mathrm{H}$ surface at $0.5-\mathrm{ML}$ coverage with $\mathrm{H}$ at one end of the hourglass site. 
used " in calibrating $\Theta$ ). The variation of $\bar{E}_{s}$ with coverage clearly exhibits two very different regimes. Between 0 and $0.5 \mathrm{ML}$ the change in $\bar{E}_{s}$ is essentially linear and amounts to only $60 \pm 5 \mathrm{meV}$, while from 0.5 to $1.0 \mathrm{ML}$ the rate of change is much greater and results in an additional $190 \pm 5-\mathrm{meV}$ shift. The existence of these two regimes is already evident in the spectra displayed in Fig. 2 and so does not depend on the details of the analysis used. However, our analytical model does show that the crossover between the two regimes takes place near the point where the unperturbed-surface intensity vanishes. A similar effect has also been seen in angle-resolved photoemission. ${ }^{12}$

We now show that these photoemission results support a recent picture of $\mathrm{H}$ chemisorption on $\mathrm{W}(110)$, which was proposed to account for the loss of (001) mirrorplane symmetry at $\sim 0.5$-ML coverage. ${ }^{8}$ In this model the $\mathrm{H}$ atoms sit at one end of the hourglass formed by four surface W atoms; see inset in Fig. 3. As $\Theta$ increases beyond the critical coverage of $0.5 \mathrm{ML}$, the top layer begins to shift along the $\langle 1 \overline{1} 0\rangle$ direction, eventually giving a global symmetry-lowering $p(1 \times 1)$ reconstruction. This proposed $\mathrm{H}$-induced structural change is very different from earlier interpretations of work function, ${ }^{13}$ thermal desorption, ${ }^{14}$ electron-stimulated desorption, ${ }^{15}$ and valence-band ${ }^{16}$ data from $\mathrm{H} / \mathrm{W}(110)$, some of which were cited as evidence for the sequential filling of two different $\mathrm{H}$ adsorption sites. Our photoemission data might also be construed as evidence for such behavior. However, if a second adsite were responsible for the observed phenomena, that site would be more weakly bound than the first (i.e., less charge is transferred) and would produce a smaller change in the average shift of the surface core level as the coverage increases. Just the opposite behavior is observed in Fig. 3, thereby ruling out the simple two-adsite picture.

By contrast, the proposed model of the $p(1 \times 1)$ reconstruction ${ }^{8}$ offers a natural explanation both for the occurrence of the crossover at $0.5 \mathrm{ML}$ and for the increased rate of change in the surface core-level binding energy beyond that point. The end of the hourglass site (as well as the long and short bridge sites) is consistent with the disappearance of the unperturbed-surface component at $0.5 \mathrm{ML}$ because it is at this coverage that all surface $W$ atoms finally have at least one nearestneighbor $\mathrm{H}$. The accelerated change in the SCS beyond that point is also easily understood. If the change in SCS over the entire coverage range were solely chemical (one binding site) with no reconstruction, then a linear change in $\bar{E}_{s}$ vs $\Theta$ would occur as in the adsorption of oxygen on this same surface. ${ }^{17}$ Such behavior is observed between 0 and $0.5 \mathrm{ML}$ in Fig. 3, where $\bar{E}_{s}$ increases by $60 \mathrm{meV}$. This implies that, in the absence of reconstruction, the net chemical shift would be +120 $\mathrm{meV}$ at saturation (indicated by the extrapolated dotdashed line in Fig. 3). Subtracting this value from the total observed shift of $250 \mathrm{meV}$ gives the additional reconstruction-induced shift of $130 \mathrm{meV}$. Thus, the interpretation of the present data is that the first $\frac{1}{2}$ monolayer is dominated by chemical bonding of the $\mathrm{H}$ atoms, and that the second $\frac{1}{2}$ monolayer has contributions from both the $\mathrm{H}$ chemical bonding and surface reconstruction.

Previously published SCS values for the clean and $\mathrm{H}$ covered W(100) and W(111) surfaces, summarized in Table I, are fully consistent with this interpretation. The more open (111) and (100) surfaces exhibit underlayer peaks, ${ }^{3-5}$ but here we consider only the outermost atoms since their spectra are the best resolved and their shifts most reliably determined. W(111), which does not reconstruct with or without $\mathrm{H}$, shows a $+170-\mathrm{meV}$ change in SCS between the clean and 3-ML-saturated surface. ${ }^{3}$ Since the $\mathrm{H}$ atoms are bridge bonded between first- and second-layer $\mathrm{W}$ atoms, ${ }^{6}$ the effective ratio of $\mathrm{H}$ to first-layer atoms at saturation is 1.5 . For $W(100)$, the highest-quality data ${ }^{3}$ show a $+95-\mathrm{meV}$ increase in SCS between the clean $c(2 \times 2)$-reconstructed surface ${ }^{18}$ and the unreconstructed $\mathrm{H}$-saturated surface at $2 \mathrm{H}$ atoms per surface $\mathrm{W} .{ }^{19}$

The absence of reconstruction on $\mathrm{W}(111)$ provides a fiducial for determining changes in the SCS due to $\mathrm{H}$ bonding alone. The observed shift of $+170 \mathrm{meV}$ for the $1.5 \mathrm{H} / 1 \mathrm{~W}$ ratio on this face predicts a chemical shift of $+113 \mathrm{meV}$ on the $1 \mathrm{H} / 1 \mathrm{~W}(110)$ surface. This value is in striking agreement with the $+120-\mathrm{meV}$ value deduced from the present data and validates our picture in which chemical-bonding effects are dominant for coverages less than $0.5 \mathrm{ML}$ on $\mathrm{W}(110)$. That the chemical shifts for the (100) and (110) surfaces are similar, despite their different $\mathrm{H}$ adsorption geometries and coverages, is not surprising in view of the fact that the $\mathrm{H}$ desorption energies at low coverages are similar for all three surfaces. ${ }^{14}$ This observation then suggests that the chemical shift for $\mathrm{W}(111)$ can be used to predict a value of $+227 \mathrm{meV}$ for

TABLE I. H-induced SCS's on low-index W surfaces (meV).

\begin{tabular}{|c|c|c|c|}
\hline Surface & $(110)$ & (111) & $(100)$ \\
\hline Clean-surface SCS ${ }^{\text {a }}$ & $-320^{b}$ & $-430^{c}$ & $-360^{d}$ \\
\hline H-saturated SCS ${ }^{a}$ & $-70^{b}$ & $-260^{c}$ & $-265^{c}$ \\
\hline $\begin{array}{l}\text { Total } \mathrm{H} \text {-induced shift } \\
\text { at saturation }\end{array}$ & +250 & +170 & +95 \\
\hline $\begin{array}{l}\text { Effective ratio of } \mathrm{H} \text { to } \\
\text { surface } \mathrm{W} \text { at saturation }\end{array}$ & 1 & 1.5 & 2 \\
\hline $\begin{array}{l}\text { H-induced chemical } \\
\text { shift at saturation }\end{array}$ & +120 & +170 & $+227^{\mathrm{e}}$ \\
\hline $\begin{array}{l}\text { Chemical shift per } \\
\text { coordinated } \mathrm{H}\end{array}$ & 120 & 113 & [1 113$]$ \\
\hline $\begin{array}{l}\text { H-induced reconstruction } \\
\text { shift at saturation }\end{array}$ & +130 & 0 & -132 \\
\hline
\end{tabular}

a With respect to bulk binding energy.

${ }^{\mathrm{b}}$ This work, $\pm 5 \mathrm{meV}$, rounded.

'Reference $3, \pm 10 \mathrm{meV}$.

${ }^{\mathrm{d}}$ Reference $5, \pm 10 \mathrm{meV}$.

${ }^{\mathrm{e}}$ Based on the W(111) shift 
the $2 \mathrm{H} / 1 \mathrm{~W}$ (100) face. Subtracting $227 \mathrm{meV}$ from the total SCS change ${ }^{3}$ of $95 \mathrm{meV}$ gives a $-132-\mathrm{meV}$ shift in going from the clean $c(2 \times 2)$-reconstructed $W(100)$ surface to the $\mathrm{H}$-saturated, unreconstructed surface, in excellent agreement with the calculated ${ }^{4}$ shift of -130 meV between a hypothetical unreconstructed clean surface and the clean $c(2 \times 2)$ phase of $W(100)$. Note that the sign of the shift is negative here because the clean surface is reconstructed and the $\mathrm{H}$-covered surface is not.

The reconstruction-induced shifts of $\sim 130 \mathrm{meV}$ for both the W(100) and W(110) surfaces can be understood within the valence-band-width framework of SCS's. ' In each case there is the same increase in effective coordination of the surface atoms compared to that of the truncated surface. On W(110), the coordination of the surface-to-subsurface-layer atoms increases from 2 to 3 , increasing the total coordination from 6 to 7 (the bulk value is 8$)$. On $\mathrm{W}(100)$, the $c(2 \times 2)$ reconstruction increases the intralayer surface coordination from 0 to 2 but reduces the effective surface-tosubsurface-layer coordination from 4 to 3 , resulting in a net change of 1 as in W(110). The increase in coordination toward the bulk value should, in both cases, increase the surface valence-band width, and shift the surface core levels toward the higher-lying bulk value, ${ }^{20}$ as observed.

In conclusion, we have determined separate chemical and reconstruction-induced shifts for the surface-atom core levels of $\mathrm{W}(110)$ produced by $\mathrm{H}$ adsorption. Comparison with $W(111)$ and $W(100)$ data leads to a selfconsistent picture in which a $\sim 120-\mathrm{meV}$ chemical shift is induced on each surface $\mathrm{W}$ per coordinated $\mathrm{H}$ atom. We further identify a $\sim 130-\mathrm{meV}$ reconstructioninduced shift for both $\mathrm{W}(110)$ and $W(100)$, which is seen as a natural extension of the intrinsic nature of the SCS. Such decomposition into chemical and structurally induced shifts should not be limited to $\mathrm{W}$, but should also be applicable whenever chemisorption triggers surface reconstruction.

The National Synchrotron Light Source at Brookhaven National Laboratory is supported by the U.S. Department of Energy, Division of Materials Sciences and Division of Chemical Sciences (DOE Contract No. DE-AC02-76CH00016).

'P. H. Citrin and G. K. Wertheim, Phys. Rev. B 27, 3176 (1983); D. Spanjaard, C. Guillot, M.-C. Desjonquères, G. Tréglia, and J. Lecante, Surf. Sci. Rep. 5, 1 (1985).

${ }^{2}$ F. J. Himpsel, B. S. Meyerson, F. R. McFeely, J. F. Morar, A. Teleb-Ibrahimi, and J. A. Yarmoff, in Photoemission and Absorption Spectroscopy of Solids and Interfaces with Synchrotron Radiation, Varenna, 1988, International School of
Physics "Enrico Fermi" (North-Holland, Amsterdam, 1989).

${ }_{3}$ J. F. van der Veen, P. Heimann, F. J. Himpsel, and D. E. Eastman, Solid State Commun. 37, 555 (1981); J. F. van der Veen et al., Solid State Commun. 40, 57 (1981); Phys. Rev. B 25, 7388 (1982).

${ }^{4}$ C. Guillot, C. Thuault, Y. Jugnet, D. Chauveau, R. Hoogewijs, J. Lecante, T. M. Duc, G. Tréglia, M. C. Desjonquères, and D. Spanjaard, J. Phys. C 15, 4023 (1982); C. Guillot et al., Solid State Commun. 50, 393 (1984).

${ }^{5}$ G. K. Wertheim, P. H. Citrin, and J. F. van der Veen, Phys. Rev. B 30, 4343 (1984).

${ }^{6}$ C. Backx, B. Feuerbacher, B. Fitton, and R. F. Willis, Phys. Lett. 60A, 145 (1977); U. A. Jayasooriya, M. A. Chesters, M. W. Howard, S. F. A. Kettle, D. B. Powell, and N. Sheppard, Surf. Sci. 93, 526 (1980).

${ }^{7}$ W. Ho, R. F. Willis, and E. W. Plummer, Phys. Rev. Lett. 40, 1463 (1978).

${ }^{8}$ J. W. Chung, S. C. Ying, and P. J. Estrup, Phys. Rev. Lett. 56, 749 (1986); M. Altman, J. W. Chung, P. J. Estrup, J. M. Kosterlitz, J. Prybyla, D. Sahu, and S. C. Ying, J. Vac. Sci. Technol. A 5, 1045 (1987).

${ }^{9}$ M. K. Debe and D. A. King, Phys. Rev. Lett. 39, 708 (1977); R. A. Barker, P. J. Estrup, F. Jona, and P. M. Marcus, Solid State Commun. 25, 375 (1978).

${ }^{10}$ D. M. Riffe, G. K. Wertheim, and P. H. Citrin, Phys. Rev. Lett. 63, 1976 (1989).

${ }^{11}$ Relative coverages are determined using the $-4-\mathrm{eV}$ zonecenter peak in the valence band, which appears as $\frac{1}{2}$ of saturation [G. B. Blanchet, N. J. DiNardo, and E. W. Plummer, Surf. Sci. 118, 496 (1982); R. H. Gaylord and S. D. Kevan (unpublished); D. A. King and D. Menzel, Surf. Sci. 40, 399 (1973)], and second-order adsorption kinetics [R. H. Gaylord, K. H. Jeong, and D. S. Kevan, Phys. Rev. Lett. 62, 2036 (1989)]. Saturation of the (100) surface corresponds to 1.0 atomic monolayer, calibrated in B. D. Barford and R. R. Rye, J. Chem. Phys. 60, 1046 (1973), in terms of the absolute H coverage of a saturated (100) surface which contains $2 \mathrm{H}$ atoms per surface $\mathbf{W}$ atom.

${ }^{12}$ Gaylord, Jeong, and Kevan (Ref. 11).

${ }^{13}$ Barford and Rye (Ref. 11).

${ }^{14}$ P. W. Tamm and L. D. Schmidt, J. Chem. Phys. 54, 4775 (1970)

${ }^{15} \mathrm{King}$ and Menzel (Ref. 11).

${ }^{16}$ B. Feuerbacher and B. Fitton, Phys. Rev. B 8, 4890 (1973); M. W. Holmes and D. A. King, Surf. Sci. 110, 120 (1981).

${ }^{17}$ D. M. Riffe, G. K. Wertheim, and P. H. Citrin (unpublished).

${ }^{18}$ Although not recognized at the time of the measurements in Refs. 3 and 4, recent results [I. K. Robinson et al., Phys. Rev. Lett. 62, 1294 (1989)] show that the room-temperature clean W(100) surface is reconstructed (as is the lowtemperature surface), albeit disordered.

${ }^{19}$ I. Stensgaard, L. C. Feldman, and P. J. Silverman, Phys. Rev. Lett. 42, 247 (1979).

${ }^{20}$ These arguments provide a qualitative insight into the origin of the reconstruction-induced shifts, and are not meant to imply a direct linear relationship between the near-neighbor coordination number and the SCS. 


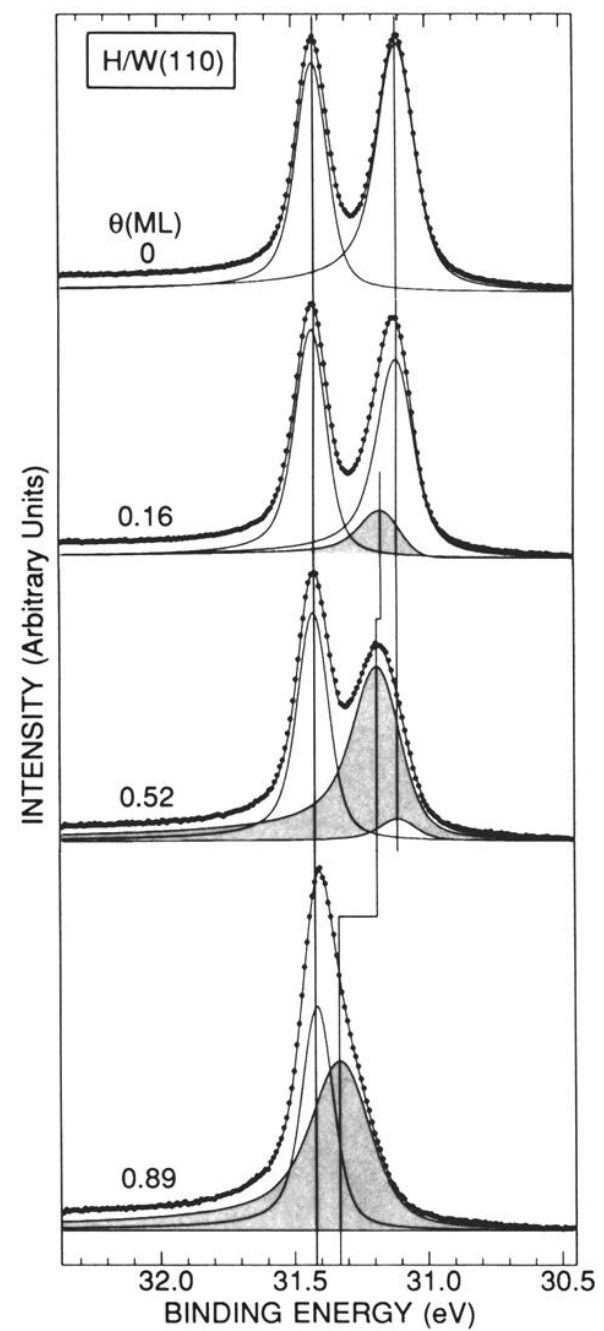

FIG. 2. Three-peak fit of $\mathrm{W} 4 f_{7 / 2}$ photoemission spectra for selected $\mathrm{H}$ coverages $\Theta$ in monolayers. Intensity of the bulk component at highest binding energy is shown equal for all fits. Bulk and unperturbed-surface line shapes and positions are held constant to their clean-surface values (see Ref. 10), but their amplitudes are allowed to vary with $\Theta$. Third component (shaded), describing the effect of the $\mathrm{H}$, is unconstrained. Solid circles are the data and the solid line is the fit. 